after sparking in this dielectric between platinum electrodes, in the same circumstances as regards voltage, current strength, and time. Consequently, there is still an uncertainty, which probably will be solved by our continued investigations.

Though the experiments, which have been mentioned here very shortly, have taken a full year, they are only the beginning of detailed investigations in different directions. Still, I feel obliged to make this communication, since I know that other investigators are repeating our sparking experiments with carbon disulphide.

A. Smrts.

Laboratory of General and Inorganic Chemistry, University of Amsterdam.

\section{Thyroid Gland and Plumage in Ghickens.}

IN a series of experiments now being carried out with Brown Leghorn chicks concerning the relation of thyroid gland to plumage characterisation, some interesting results are already apparent in the thyroidectomised females. The operation was carried out when the birds were 6 weeks old, and 3 weeks after

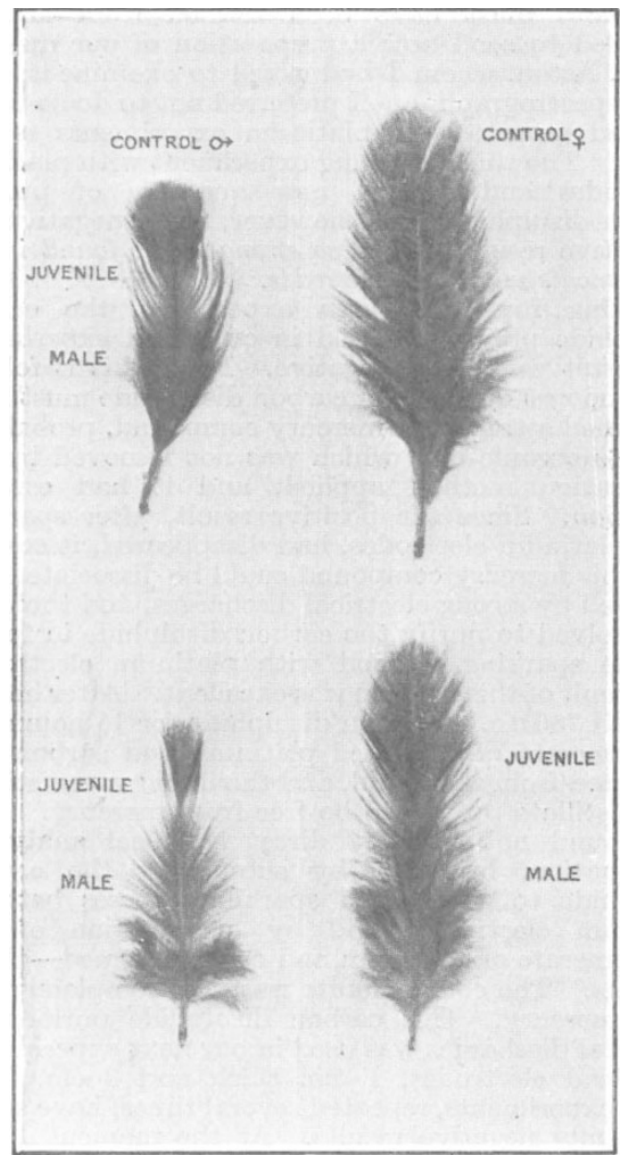

Fit. 1-Wing contour feathers from Brown Lewhorn ehicks 10 weeks old. Upper fierures, controls;

the operation the effect on the plumage became visible. Changes in coloration first appear in the contour feathers of the wing, shoulder and cape, the whole forming a continuous arc of brightly coloured feathers from one wing edge to the other when the bird is examined with the wings outstretched. Examination of control male chicks at the same time showed a similar continuous band of brightly coloured feathers replacing the juvenile plumage in these areas, while no such band was present in the control femalos. In the region of the saddle, brightly coloured feathers were also observed in the thyroidectomised females and in control males, while being absent in control females.

When the feathers were sufficiently grown to determine their shape, it was seen that the majority of these brightly coloured feathers have the blunt tip characteristic of the juvenile fonther. At a short distance from the tip, however, the shape changes abruptly, and in the proximal portion the feather is very similar to the typical male feather from the same regions, $i . e$. it is hoavily fringed and tapers almost to a point at the junction with the distal juvenile portion. This condition is also found in feuthers from control males of approximately the same age (Fig. 1). The coloration of the male-like feathers in the thyroidectomised females, whilo markedly differing from fomale colouring, is not quite so deep as that of the feathers from control males.

It would appear thus that the removal of the thyroid gland from Brown Leghorn female chicks leads in the first place to the assumption of plumage of a type approaching in colour and form that of the male. These results are of special interest in view of the rocent work on the effect of thyroid feeding on the plumage of the fowl. A. W. Greenwoon.

Animal Breeding Researoh Department, University of Edinburgh.

\section{Ultrasonic Stationary Waves.}

THe observations described in the striking experiment of Hubbard and Loomis (NATURE, Aug. 6, 1927, p. 189) are another example of the important conclusions which may be derived from a study of the interesting phenomonon of ultrasonic stationary waves. Velocities of sound in various liquids were determined here by the ultrasonic stationary wave method somo years ago ; some of these results have already been published (Trans. Roy. Soc. Can., 3, 141 ; $1923 ; 159,191,197 ; 1925 ; 79 ; 1927)$; others were reported to the Canadian Research Council (Report, Boyle and Morgan, 1924). The 'detector' of the standing waves in these experiments was "nodal dust figures,' something like the figures in a Kundt's tube, but less precision was claimed for the results than is claimed by the authors above.

Pierce also carried out very precise experiments on the volocity of sound in gases (Proc, Amer, Acad., 60, 6,271 ; 1925) by the ultrasonic mothod, the detector of the standing waves in his experiments boing a milli- or micro-ammeter in the associated grid-circuit of the electric generating tube. In fact, Hubbard and Loomis's experiment does for liquids what Pierce's did for gases, with the exception that their standing wave indicator is a neon tube instead of a milliammeter.

The purpose of this note is to point out that the prosence of these ultrasonic stationary waves in a liquid can easily bo demonstrntod and visualised by making use of another phenomenon, namely, that of ultrasonic cavitation, or the production of bubbles in the liquid by the waves themselves. In our work in this laboratory nodal layers of bubbles, a half wave. length apart, have been produced in a tank of liquid between an ultrasonic generator and a reflector ; and some months ago Messrs. Taylor and Sproule arranged an apparatus for ultrasonic waves in which a bell-jar

No. 3022, VoL. 120] 